\title{
Investigation of the prompt neutron emission mechanism in low energy fission of ${ }^{235,233} U\left(n_{\text {th }}, f\right)$ and ${ }^{252} \mathrm{Cf}(\mathrm{sf})$
}

\author{
A.S. Vorobyev ${ }^{1, \text { a }}$, O.A. Shcherbakov ${ }^{1}$, A.M. Gagarski ${ }^{1}$, G.V. Val'ski ${ }^{1}$, and G.A. Petrov ${ }^{1}$ \\ ${ }^{1}$ PNPI, Neutron Research Department, 188300 Gatchina, Leningrad district, Russia
}

\begin{abstract}
A series of experiments has been performed to measure prompt neutron angular and energy distributions from thermal neutron-induced fission of ${ }^{235,233} \mathrm{U}$ in correlation with the fission fragments. These distributions have been analyzed with the assumption of neutron isotropic emission from accelerated fission fragments. The performed analysis demonstrates that all obtained results can be described within 5\% accuracy using this assumption. This discrepancy is approximately constant and doesn't depend on fragment mass and the total kinetic energy (TKE). Some minor peculiarities of angular distribution may be interpreted as a result of anisotropy of the fission neutron angular distribution in the fragment center-of-mass system.
\end{abstract}

\section{Introduction}

It is well known that the main part of prompt fission neutrons are emitted from the excited fragments accelerated in the Coulomb field of nuclei. At that, a part of prompt neutrons originated due the emission mechanism of another nature is varied from $30 \%$ down to the total absence of such neutrons [1]. At the same time, there are only a few experimental works which contain numerical information that can be used for analysis of the experimental data. Using the data of these works, it was ascertained [2] that about 0.4 neutrons per fission can not be described within the framework of a simple evaporation model. The energy spectra of such neutrons consist of two components with average energies $0.9 \mathrm{MeV}$ and $3 \mathrm{MeV}$. According to the author's opinion, the most likely mechanism of emission of these additional neutrons is emission from fissioning nucleus at the stage of its descent to the scission point (the so-called "pre-scission" neutrons) [3]. The direct answer to the question on properties of these additional neutrons could be obtained from the measurements of angular and energy distributions of prompt fission neutrons in the laboratory system in the correlation with the fission fragment characteristics. The measurements of this type were performed recently at the PNPI RAS. In the present paper we present first results of these measurements.

\section{Data processing}

The fission fragments and prompt neutrons time-of-flights were measured simultaneously for 11 fixed angles, $\theta$, between the axis of neutron detector and normal to the stop MWPD surface (coming through its center) in the range from $0^{\circ}$ to $180^{\circ}$ in $18^{\circ}$ intervals (see Fig. 1).

The prompt neutrons were detected using two stilbene crystal detectors positioned at a $90^{\circ}$ angle between their respective axes at a distance of $\sim 48 \mathrm{~cm}$ from the fissile target. The neutron registration threshold was 150-200 keV. To separate events corresponding to neutrons and $\gamma$-quanta, a double

\footnotetext{
a e-mail: alexander.vorobyev@pnpi.spb.ru
}

This is an Open Access article distributed under the terms of the Creative Commons Attribution-Noncommercial License 3.0, which permits unrestricted use, distribution, and reproduction in any noncommercial medium, provided the original work is properly cited. 


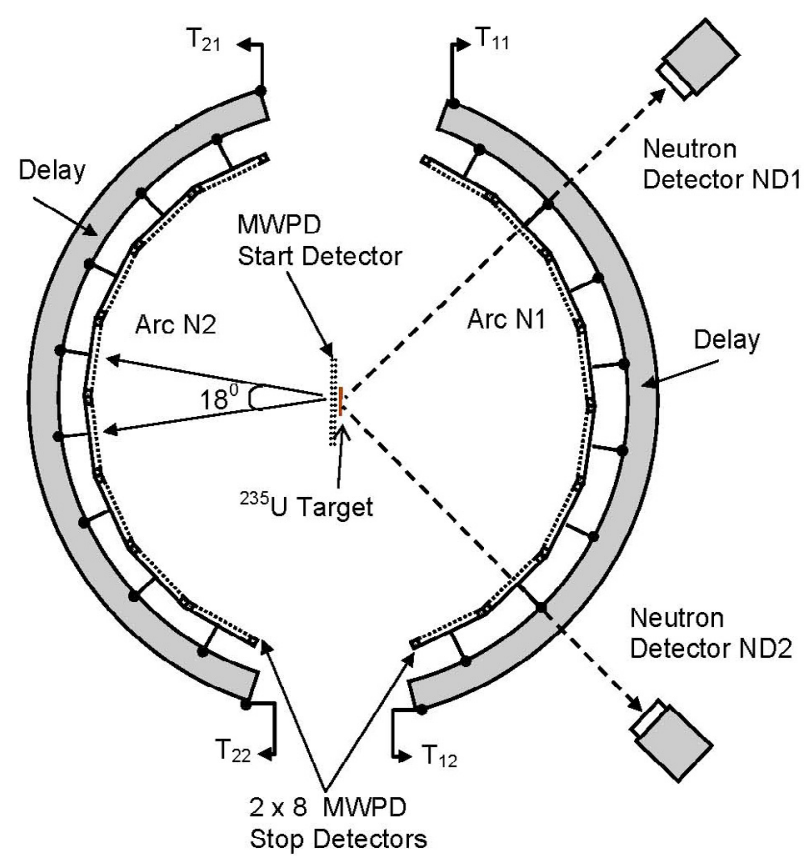

Fig. 1. Schematic view of the experimental set-up (no scale).

discrimination by the pulse shape and time-of-flight was applied. The full time uncertainties were defined from FWHM of the "fragment - $\gamma$-quantum" coincidence curve which was equal to $1.2 \mathrm{~ns}$.

The fission fragments were detected by multi-wire proportional detectors (MWPDs) in conjunction with the TOF technique. A detail description of the experimental set-up and some preliminary results can be found in Ref. $[4,5]$.

During data processing, the following corrections were taken into account for: time uncertainties in TOF measurements, neutron detector background, neutron detector efficiency, angular and neutron energy resolution, fragment transmission of the start and stop MWPDs, the fragment detector efficiency and for incomplete separation of light and heavy group of fission fragments, normalization correction connected to the fact that in the measurements we have the experimental histogram distributions instead of continuous distributions. To determine the neutron detectors effeciencies, the additional measurements of prompt neutron angular and energy distribution have been performed recently for ${ }^{252} \mathrm{Cf}(s f)$ at the same experimental conditions and set-up (the only difference was an opacity of the target backing for ${ }^{252} \mathrm{Cf}$ fission fragments). So the neutron detector efficiency was determined for each neutron detector independently as the ratio of the obtained total prompt neutron spectrum of ${ }^{252} \mathrm{Cf}$ to a reference standard spectrum [6].

\section{Analysis}

It is well established that the prompt neutrons in low energy fission are emitted mainly from fully accelerated fragments and the yield of neutrons with other emission mechanism, so called "scission" neutrons, may be no more than $30 \%$ of the total prompt neutron yield in low energy fission. The contribution of "scission" neutrons is determined as difference between experimentally observable variables in the laboratory system and those calculated using known neutron spectra in the center-ofmass system on the basis of the assumption that neutrons are emitted only from accelerated fragments. As the prompt neutron energy spectra in the center-of-mass system of light and heavy fragments, the Maxwellian or Weisskopf spectra are usually used. The parameters of these spectra are adjusted so as to show the best fit of available experimental data. 
In our analysis the angular and energy spectra of the prompt fission neutrons emitted by accelerated fragments are calculated using experimental data for $0^{\circ}, 18^{\circ}$ and $36^{\circ}$ angles relative to the fission direction [5]. Thus the calculated spectra are free of any parameters concerning the prompt neutron spectra in the center-of-mass system (the number of neutrons emitted by heavy and light fragments, the neutron spectrum shapes and so on). During this calculation, as a first approximation, it was assumed that prompt neutrons are emitted by two fragments with average mass and kinetic energy, since, as it was shown for ${ }^{252} \mathrm{Cf}(s f)$ [7], it has a minor influence on the total neutron energy spectrum. As the average energy per nucleon for light and heavy fragments, the specific energy obtained in our measurements was used. These values were taken as $\left\langle E_{L}\right\rangle=1.046,1.025 \mathrm{MeV}$ and $\left\langle E_{H}\right\rangle=0.471,0.476 \mathrm{MeV}$ for ${ }^{233,235} \mathrm{U}\left(n_{t h}, f\right)$, respectively. For ${ }^{252} \mathrm{Cf}(s f)$ these values were taken from literature [2] and were equal to $\left\langle E_{L}\right\rangle=0.951 \mathrm{MeV}$ and $\left\langle E_{H}\right\rangle=0.542 \mathrm{MeV}$.

\section{Results and discussion}

\subsection{Angular and energy distributions}

The number of fission neutrons and their average energy for fixed angles in the laboratory system (obtained experimentally and calculated using an assumption about neutron emission from accelerated fragments) are shown at the top of Figs. 2, 3. Both experimental and model neutron spectra have been compared in $0.2-10 \mathrm{MeV}$ energy range. The errors of the obtained experimental data are comparable with the point's size. In these figures, the experimental results of Skarsvag and Bergheim for ${ }^{235} \mathrm{U}$ [8] and of Bowman et al. for ${ }^{252} \mathrm{Cf}$ [9] are also shown. These authors came to a conclusion that about $10-15 \%$ of neutrons are emitted during the fission process itself. In our case, on the whole, the calculated model energy and angular distributions agree rather well with the experimentally obtained distributions.

However, there is a minor distinction which is most clearly demonstrated at the bottom of Figs. 2, 3 where the angular dependence of the ratios of experimentally obtained neutron yield and average energy to calculated values are shown. The error "corridors" presented in these Figs are the standard deviation of ratio obtained for each cycle of measurements to the average ratio obtained for all cycles of measurements. Special attention must be given to the fact that the model calculation gives overestimated values of fission neutron yield and average energy as compared with the experiment for all

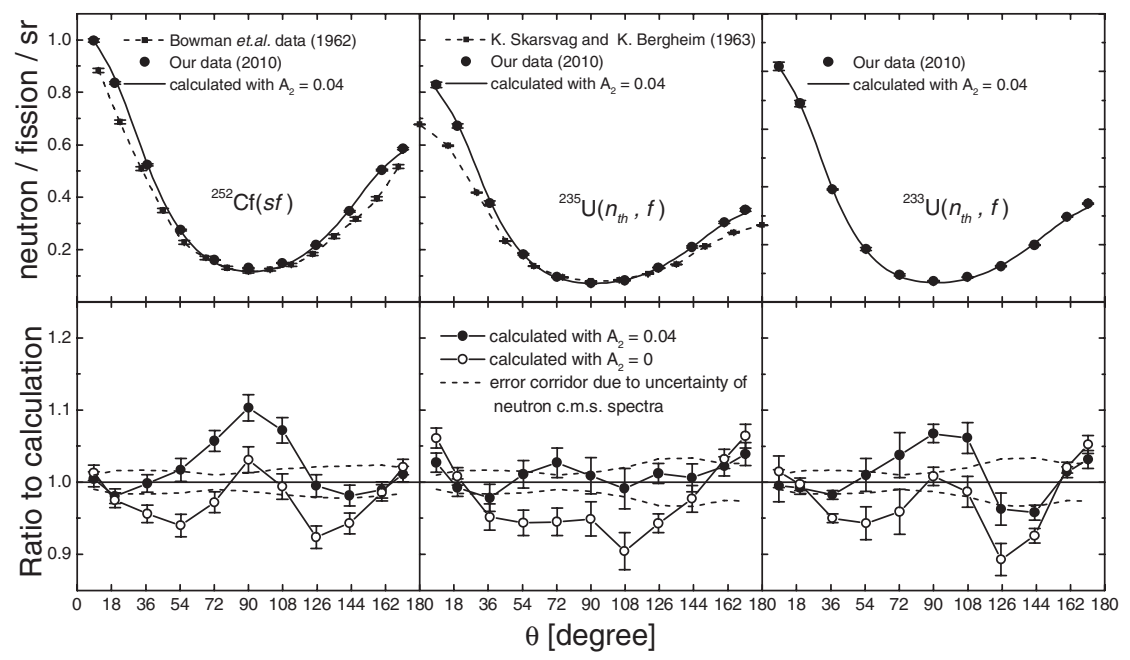

Fig. 2. Fission neutron yield as a function of the angle between neutron flight direction and the direction of motion of the light fragment. 


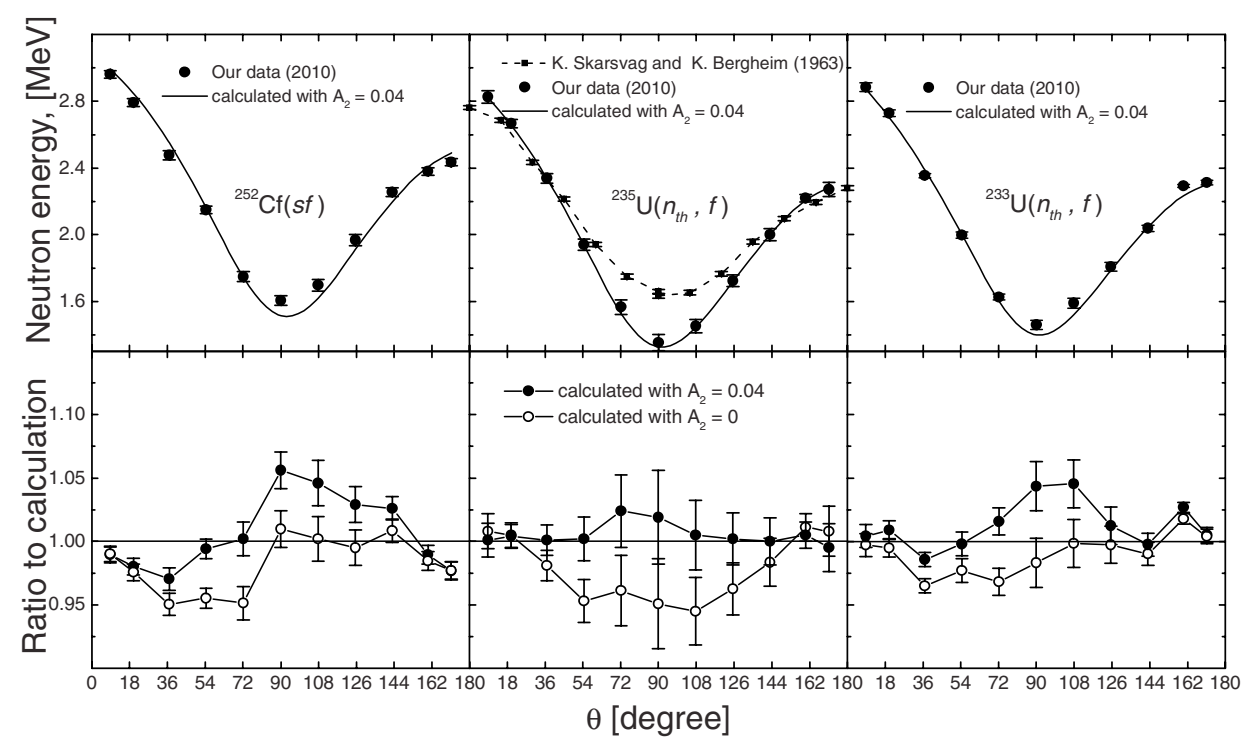

Fig. 3. Angular dependence of the average neutron emission energy in the laboratory system.
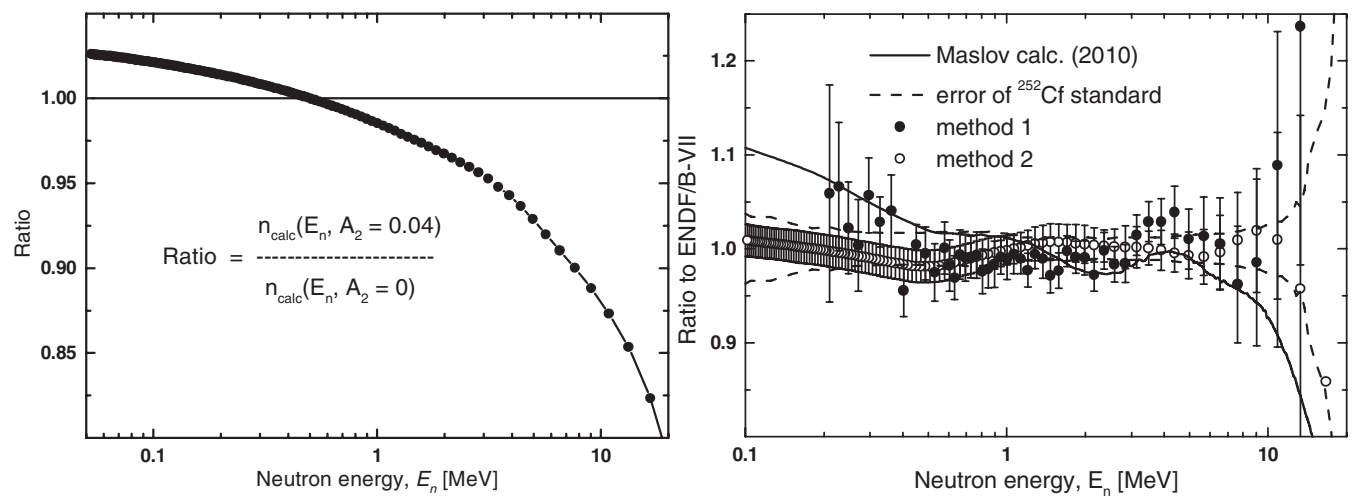

Fig. 4. Left: Ratio spectra calculated with to without anysotropy; Right: the PFNS of ${ }^{235} \mathrm{U}\left(n_{t h}, f\right)$ as a ratio to ENDF/B-VII evaluation, (log scale).

measured angles. Our model calculation shows that such discrepancy may be related to the presence of anisotropy of the fission neutron angular distribution in the center-of-mass system.

Introduction of anisotropy with $\mathrm{A}_{2}=0.04 \div 0.06$ into the model calculation improves agreement with obtained experimental data. At this, there is some surplus of measured yield over calculated at angles near $90^{\circ}$. Under the assumption that these "additional" neutrons are emitted isotropically in the laboratory system, their yield may be deduced as not more than $5 \%, 4 \%$ and $3 \%$ of the total neutron yield for ${ }^{252} \mathrm{Cf}(s f),{ }^{233} \mathrm{U}\left(n_{t h}, f\right)$ and ${ }^{235} \mathrm{U}\left(n_{t h}, f\right)$ respectively.

\subsection{Total prompt neutron spectrum}

The total prompt neutron spectrum, PFNS, (integrated over all angles in the laboratory system, method 1) for all investigated isotopes agrees within experimental errors with one calculated (method 2$)$ in the assumption of anisotropy $\left(\mathrm{A}_{2}=0.04\right)$ of the fission neutron angular distribution in the center-of-mass system of fragments (Fig. 4, right). It should be noted that calculated shape and 
EFNUDAT Workshop “Measurements and Models of Nuclear Reactions”, Paris, 2010

Table 1. Average total prompt neutron multiplicity.

\begin{tabular}{|c|c|c|c|c|}
\hline \multirow{2}{*}{ Target } & \multicolumn{3}{|c|}{$v_{\text {prompt }}$ (neutron / fission) } \\
\cline { 2 - 3 } & \multicolumn{2}{|c|}{ Calculated (method 2) } & \multirow{2}{*}{ Experiment (method 1) } & \multirow{2}{*}{ ENDF/B-VII } \\
\cline { 2 - 3 } & $\mathrm{A}_{2}=0$ & $\mathrm{~A}_{2}=0.04$ & & 3.7590 \\
\hline${ }^{252} \mathrm{Cf}(s f)$ & 3.86 & 3.73 & $3.77 \pm 0.02$ & 2.421 \\
\hline${ }^{235} \mathrm{U}\left(n_{t h}, f\right)$ & 2.56 & 2.45 & $2.44 \pm 0.05$ & 2.4894 \\
\hline${ }^{233} \mathrm{U}\left(n_{t h}, f\right)$ & 2.60 & 2.48 & $2.54 \pm 0.06$ & \\
\hline
\end{tabular}
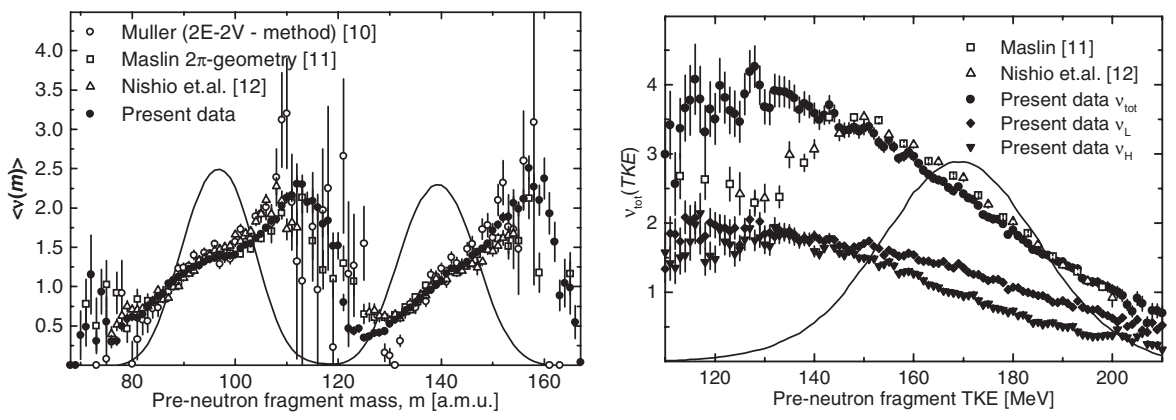

Fig. 5. The neutron yield as a function of pre-neutron fragment mass and TKE together with pre-neutron fission fragment mass and TKE distribution obtained by these measurements (for ${ }^{235} \mathrm{U}\left(n_{t h}, f\right)$.

average prompt neutron multiplicity are dependent on the value of anysotropy included. For example, this effect is demonstrated in the left of Fig. 4 for ${ }^{235} \mathrm{U}\left(n_{t h}, f\right)$ as ratio spectra calculated with inclusion anysotropy to without it.

In Table 1 the calculated average prompt neutron multiplicities are presented in comparision with our experimental data obtained by summation over angles. It is seen that the calculation performed using experimental data obtained for small angles relative to the fission fragment direction reproduces the total prompt neutron spectrum, both the shape and the average multiplicity.

\subsection{Neutrons and fission fragments correlation}

To obtain surely the yield and to ascertain an emission mechanism of these neutrons, it is necessary to perform a more careful analysis of measured angular and energy distributions of ${ }^{233,235} \mathrm{U}\left(n_{t h}, f\right)$. It can be done in the same manner as we used, but in this case the transformation of neutron spectrum in the laboratory system (at small angles relative to fragments direction) to the center-of-mass system should be performed for each fission fragment (FF) fixed mass and energy. At present, such analysis is in progress. The average neutron multiplicity as a function of pre-neutron fragment mass and total kinetic energy (TKE) for ${ }^{235} \mathrm{U}\left(n_{t n}, f\right)$ are presented in Fig. 5. A good agreement is observed between data obtained by this experiment and other authors [10-12]. Some discrepancy is seen in low TKE region, where for decreasing TKE the neutron yield increases approximately linear in our case while it decreases in other work. This difference needs an explanation. Probably, that is simply due to the scattered events since at these energies the count rate is extremely low and a few scattered events will have a big influence.

The neutron yield at $90^{\circ}$ relative to FFs direction in the laboratory system as function of fission fragment properties was calculated to examine the model (neutrons are emitted isotropically by accelerated fragments) used to calculation of neutron yield presented in Fig. 5. The measured dependencies look like the calculated ones (Fig. 6). It is seen that the model calculation gives overestimated values of fission neutron yield as for two fragment approximation case (see subsection 4.1). Since the inclusion 

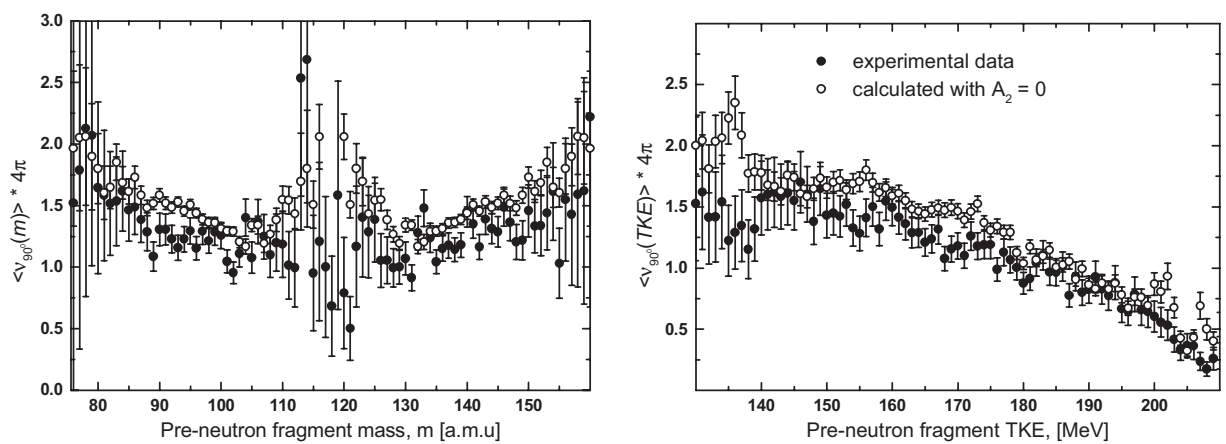

Fig. 6. The comparison of the measured neutron yield at $90^{\circ}$ relative to FFs direction as a function of pre-neutron fragment mass and TKE with calculated one. For convenience the neutron yield was multiplied by $4 \pi$ (one cycle of measurements for $\left.{ }^{235} \mathrm{U}\left(n_{t h}, f\right)\right)$.

of neutron anisotropy into calculation reduces the calculated prompt neutron yield by about $10 \%$, there is a very good agreement within statistical errors (presented as error bars in Fig. 6) between measured and calculated neutron yield at $90^{\circ}$ relative to FFs in the laboratory system. It should be noted that both measured yield and calculation presented in Fig. 6 are the result of a single measurement cycle. To make a quantitative conclusion about a part of "scission" neutrons it needs to apply procedure analogous mentioned above for all measured cycles.

\section{Conclusions}

The prompt fission neutron energy spectra have been measured for ${ }^{235,233} \mathrm{U}\left(n_{t h}, f\right)$ and $\operatorname{Cf}(s f)$ at 11 fixed angles between the neutron and light fragment directions in the range from $0^{\circ}$ to $180^{\circ}$. The comparison of experimentally obtained angular and energy distributions of prompt neutrons and calculated ones on the base of neutron emission from accelerated fission fragments enables: 1) to estimate the contribution of "scission" neutrons as not to exceed 5\% of total neutron yield in an assumption of isotropic emission in the laboratory system; 2) to conclude that the angular anisotropy of the neutron emission in the fragment center-of-mass system, $\mathrm{A}_{2} \sim 0.04\left(\mathrm{~b}=3 \mathrm{~A}_{2} /\left(2-\mathrm{A}_{2}\right)\right)$, should be included into any calculation of prompt neutron properties in the nuclear fission.

Now we are doing more careful analysis of the obtained angle-energy distributions to determine the dependence of main characteristics of "scission" neutrons on fragment mass and kinetic energy.

In the future we are planning to carry out the same experiment for ${ }^{239} \mathrm{Pu}\left(n_{t h}, f\right)$.

We wish to thank D.O. Krinitsin, L.S. Falev, Yu.S. Pleva and V.E. Sokolov for help in adjustment of the equipment and conducting the experiments, T.E. Kuz'mina and S.M. Soloviev from Khlopin Radium Institute for producing the ${ }^{233,235} \mathrm{U}$ targets. The authors are very grateful to I.S. Guseva for helpful discussions. The help of V.I. Petrova and T.A. Zavarukhina is also highly appreciated.

\section{References}

1. G.A. Petrov, AIP Conference Proceedings, 798, Issue 1, 205 (2005).

2. N.V. Kornilov, A.B. Kagalenko, F.-J. Hambsch, Sov. J. Nucl. Phys. 64, 1462 (2001); N.V. Kornilov, A.B. Kagalenko, S.V. Poupko, et al., Nucl. Phys. A 686, 187 (2001).

3. N.V. Kornilov, F.-J. Hambsch and A.S. Vorobyev, Nucl. Phys. A 789, 55 (2007).

4. G.V. Val'ski, A.M. Gagarski, I.S. Guseva, et al., PNPI Preprint-2546 (Gatchina, 2003).

5. A.S. Vorobyev, O.A. Shcherbakov, et al., Nucl. Instr. and Meth. A 598, 795 (2009). 
EFNUDAT Workshop "Measurements and Models of Nuclear Reactions", Paris, 2010

6. C.W. Reich, W. Mannhart, T. England, ENDF/B-VII.

7. D.G. Madland, IAEA Report INDC(NDS) - 251, 201 (Vienna, 1991).

8. K. Skarsvag and K. Bergheim, Nucl. Phys. 45, 72 (1963).

9. H.R. Bowman, J.C.D. Milton et al., Phys. Rev. 126, 2120 (1962).

10. R. Muller, A.A. Naqvi, F. Kappeler, and F. Dickmann, Phys. Rev. C29, 885 (1984).

11. E.E Maslin, A.L. Rodgers, and W.G.F. Core, Phys. Rev. 164, 1520 (1967).

12. K. Nishio, Y. Nakagome, H. Yamamoto, I. Kimura, Nucl. Phys. A632, 540 (1998). 